\title{
Calcium-46 Chloride Aqueous Solution
}

National Cancer Institute

\section{Source}

National Cancer Institute. Calcium-46 Chloride Aqueous Solution. NCI Thesaurus. Code C85474.

An orally bioavailable aqueous solution containing the chloride salt of the radioisotope calcium-46 (46Ca) with phosphate-binding and radioisotopic activities. Upon administration of calcium- 46 chloride aqueous solution, calcium- 46 is preferentially taken up by osteoblasts, which generate mineralized osteoid containing calcium. Calcium-46 accumulation and turnover in bone can be measured with bone scintig raphy and urinary isotope excretion testing. 